\title{
SOME TAUBERIAN THEOREMS CONCERNING
}

\section{$\left(S^{*}, \mu\right)$ TRANSFORMATIONS}

\section{DANY LEVIATAN}

(Received July 30, 1968)

1. Introduction. The regular series to sequence $\left(S^{*}, \mu\right)$ transform of a series $\sum_{i=0}^{\infty} a_{i}$ is defined as follows :

$$
S_{n}^{*}(\beta)=\sum_{i=0}^{\infty} a_{i} \sum_{k=i}^{\infty}\left(\begin{array}{c}
k+n \\
n
\end{array}\right) \int_{0}^{1}(1-t)^{k} t^{n+1} d \beta(t), n \geqq 0,
$$

where $\beta(t)$ satisfies

(1. 2) $\beta(t)$ is of bounded variation in $[0,1], \beta(1)-\beta(0+)=1$ and $\beta(1)=\beta(1-)$.

The series to sequence $\left(S^{*}, \mu\right)$ transformation is the series to sequence analogues of the sequence to sequence $\left(S^{*}, \mu\right)$ transformation defined by Ramanujan [7] $\S 4$. We shall be interested in finding Tauberian estimates of the following form. For a series $\sum_{i=0}^{\infty} a_{i}$ denote $s_{n}=\sum_{i=0}^{n} a_{i}$, then what is the best possible constant $A$ satisfying

$$
\limsup _{\lambda \rightarrow \infty}\left|S_{n(\lambda)}^{*}(\beta)-s_{m(\lambda)}\right| \leqq A \limsup _{n \rightarrow \infty}\left|n a_{n}\right|
$$

where $n(\lambda), m(\lambda)$ are given functions assuming integral values only, and all series $\sum_{i=0}^{\infty} a_{i}$ satisfying the Tauberian condition

$$
\limsup _{n \rightarrow \infty}\left|n a_{n}\right|<\infty
$$

What is the best constant $B$ satisfying

$$
\limsup _{\lambda \rightarrow \infty}\left|S_{n(\lambda)}^{*}(\beta)-S_{m(\lambda)}^{*}(\gamma)\right| \leqq B \limsup _{n \rightarrow \infty}\left|n a_{n}\right|
$$

where $\gamma(t)$ is another function satisfying (1.2), $n(\lambda), m(\lambda)$ are as before and 
$\sum_{i=0}^{\infty} a_{i}$ satisfies (1.3). What is the best constant $C$ satisfying

$$
\limsup _{n \rightarrow \infty}\left|S_{n(\lambda)}^{*}(\beta)-s_{m(\lambda)}\right| \leqq C \limsup _{\lambda \rightarrow \infty}\left|b_{n}\right|
$$

where $n(\lambda), m(\lambda)$ are as before, $b_{n}=\left(a_{1}+2 a_{2}+\cdots+n a_{n}\right) /(n+1) n \geqq 1$ and our series satisfies the Tauberian condition weaker than (1.3),

$$
\limsup _{n \rightarrow \infty}\left|b_{n}\right|<\infty .
$$

In order to simplify the notation we write instead of (1.2),

(1.5) $\beta(t)$ is of bounded variation in $[0,1], \beta(0+)=0$ and $\beta(1)=\beta(1-)=1$.

We shall restrict ourselves to function $\beta(t)$ satisfying

$$
\beta(0)=0, \quad \int_{0}^{1} x^{-1}|\beta(x)| d x<\infty, \int_{0}^{1}(1-x)^{-1}|1-\beta(x)| d x<\infty .
$$

By inspecting the Tauberian estimates obtained in the following sections one sees that the condition (1.6) is necessary in order to obtain finite constants $A, B$, or $C$ and thus not much of a restriction. Recently the first problem was discussed by $\mathrm{S}$. Sherif [8] under an additional assumption that $\beta(t)$ is non-decreasing in $[0,1]$.

\section{Main results.}

THEOREM 1. For a series $\sum_{i=0}^{\infty} a_{i}$ satisfying (1.3) and a function $\beta(t)$ satisfying (1.5) and (1.6) we have for each $q, 0<q<\infty$ and any two functions $n(\lambda) \rightarrow \infty m(\lambda) \rightarrow \infty$ assuming integral values only and satisfying $m(\lambda) / n(\lambda) \rightarrow q$ as $\lambda \rightarrow \infty$,

$$
\limsup _{\lambda \rightarrow \infty}\left|S_{n(\lambda)}^{*}(\beta)-s_{m(\lambda)}\right| \leqq A_{q} \limsup _{n \rightarrow \infty}\left|n a_{n}\right|
$$

where

$$
A_{q}=\int_{0}^{1 /(q+1)} \frac{|\beta(x)|}{x(1-x)} d x+\int_{1 /(q+1)}^{1} \frac{|1-\beta(x)|}{x(1-x)} d x
$$

The constant $A_{q}$ is the best possible in the following sense. There exists a series $\sum_{i=0}^{\infty} a_{i}$ satisfying (1.3) and the members of inequality (2.1) are equal. 
Theorem 1 for a non-decreasing $\beta(t)$ was proved by S.Sherif [8]. For the function $\beta(t)=0$ for $0 \leqq t<1-\alpha$ and $\beta(t)=1$ for $1-\alpha \leqq t \leqq 1(0<\alpha<1)$ the series to sequence $\left(S^{*}, \mu\right)$ transform of a series $\sum_{i=0}^{\infty} a_{i}$ is the sequence to sequence $S_{\alpha}$ transform of the sequence $s_{n}=\left\{\sum_{i=0}^{n} a_{i}\right\}(n \geqq 0)$ defined by MeyerKönig [5]. Theorem 1 for the $S_{\alpha}$ transformation was proved by Biegert [2].

THEOREM 2. For a series $\sum_{i=0}^{\infty} a_{i}$ satisfying (1.3) and functions $\beta(t)$ and $\gamma(t)$ satisfying (1.5) and (1.6) we have for each $q, 0<q<\infty$ and any two functions $n(\lambda) \rightarrow \infty m(\lambda) \rightarrow \infty$ assuming integral values only and satisfying $m(\lambda) / n(\lambda) \rightarrow q$ as $\lambda \rightarrow \infty$,

$$
\limsup _{\lambda \rightarrow \infty}\left|S_{n(\lambda)}^{*}(\beta)-S_{m(\lambda)}^{*}(\gamma)\right| \leqq B_{q} \limsup _{n \rightarrow \infty}\left|n a_{n}\right|
$$

where

$$
B_{q}=\int_{0}^{1} \frac{\left|\beta\left(\frac{t}{q-(q-1) t}\right)-\gamma(t)\right|}{t(1-t)} d t
$$

The constant is the best possible in the following sense. There exists a series $\sum_{i=0}^{\infty} a_{i}$ satisfing (1.3) and the members of inequality (2.3) are equal.

REMARK. We note that the constant $B_{q}$ is better than estimates we could obtain by Theorem 1 , by introducing a function $p(\lambda)$ assuming integral values only and such that $p(\lambda) / m(\lambda) \rightarrow a, 0<a<\infty$ and estimating $\left|S_{n}^{*}(\beta)-S_{m}^{*}(\gamma)\right|$ by

$$
\left|S_{n}^{*}(\beta)-S_{m}^{*}(\gamma)\right| \leqq\left|S_{n}^{*}(\beta)-s_{p}\right|+\left|s_{p}-S_{m}^{*}(\gamma)\right|
$$

The computations are left to the reader.

THEOREM 3. For a series $\sum_{i=0}^{\infty} a_{i}$ satisfying (1.4) and a continuous function $\beta(t)$ satisfying (1.5) and (1.6), we have for each $q, 0<q<\infty$ and any two functions $n(\lambda) \rightarrow \infty, m(\lambda) \rightarrow \infty$ assuming integral values only and satisfying $m(\lambda) / n(\lambda) \rightarrow q$ as $\lambda \rightarrow \infty$,

$$
\limsup _{\lambda \rightarrow \infty}\left|S_{n(\lambda)}^{*}(\beta)-s_{m(\lambda)}\right| \leqq C_{q} \limsup _{n \rightarrow \infty}\left|b_{n}\right|
$$


where

$$
\begin{gathered}
C_{q}=1+\int_{0+}^{1 /(1+q)} \frac{1-t}{t}\left|d\left[\frac{t}{1-t} \beta(t)\right]\right|+\int_{1 /(1+q)}^{1-} \frac{1-t}{t}\left|d\left[\frac{t}{1-t}(1-\beta(t))\right]\right| \\
\left(\text { where } \int_{0+}=\lim _{\rho \downarrow 0} \int_{\rho} \text { and } \int^{1-}=\lim _{\eta \uparrow 1} \int^{\eta}\right) .
\end{gathered}
$$

The constant $C_{q}$ is the best possible in the following sense. There exists a series $\sum_{i=0}^{\infty} a_{i}$ satisfying (1.4) and the members of inequality (2.5) are equal.

The following is an immediate consequence of Theorem 3.

COROLlARY. If, in addition to the assumption of Theorem 3 , the functions $\frac{t}{1-t} \beta(t)$ and $\frac{t}{1-t}(1-\beta(t))$ are non-decreasing for $0<t<1$, then

$$
C_{q}=\int_{0}^{1 /(1+q)} \frac{\beta(x)}{x(1-x)} d x+\int_{1 /(1+q)}^{1} \frac{1-\beta(x)}{x(1-x)} d x+2 \beta\left(\frac{1}{1+q}\right)
$$

Inasmuch as $0 \leqq \beta(t) \leqq 1$ for $0 \leqq t \leqq 1$ we have

$$
C_{q}=A_{q}+2 \beta\left(\frac{1}{1+q}\right)
$$

THEOREM 4. For a series $\sum_{i=0}^{\infty} a_{i}$ satisfying (1.4) and a function $\beta(t)$ satisfying (1.5), (1.6) and $\beta(t)=\frac{1}{2}[\beta(t+)+\beta(t-)]$ for $0<t<1$, we have for each $q, 0<q<\infty$,

$$
\limsup _{n \rightarrow \infty}\left|S_{n}^{*}(\beta)-s_{[n q]}\right| \leqq C_{q} \limsup _{n \rightarrow \infty}\left|b_{n}\right|
$$

where $C_{q}$ is defined by (2.6) and is the best constant possible in the following sense. There exists a series $\sum_{i=0}^{\bar{c}} a_{i}$ satisfying (1.6) and the members of inequality (2.7) are equal. 


\section{Proofs of Theorem 1 and 2.}

PROOF OF THEOREM 1. First we prove that whenever the series $\sum_{i=0}^{\infty} a_{i}$ satisfies (1.3) and $\beta(t)$ satisfies (1.5) and (1.6), then $S_{n}^{*}(\beta)$ exists for every $n \geqq 0$. It follows immediately that for $0 \leqq t \leqq 1$ and $i>0$

$$
\begin{aligned}
\frac{d}{d t} \sum_{k=i}^{\infty}\left(\begin{array}{c}
k+n \\
n
\end{array}\right)(1-t)^{k} t^{n+1} & =\frac{d}{d t}\left[1-\sum_{k=0}^{i-1}\left(\begin{array}{c}
k+n \\
n
\end{array}\right)(1-t)^{k} t^{n+1}\right] \\
& =-i t^{-1}(1-t)^{-1}\left(\begin{array}{c}
i+n \\
n
\end{array}\right)(1-t)^{i} t^{n+1}
\end{aligned}
$$

whence

$$
\sum_{k=i}^{\infty}\left(\begin{array}{c}
k+n \\
n
\end{array}\right)(1-t)^{k} t^{n+1}=i \int_{t}^{1} u^{-1}(1-u)^{-1}\left(\begin{array}{c}
i+n \\
n
\end{array}\right)(1-u)^{i} u^{n+1} d u
$$

By Beppo-Levi's theorem for every $i \geqq 0$

$$
\begin{aligned}
\sum_{k=i}^{\infty}\left(\begin{array}{c}
k+n \\
n
\end{array}\right) \int_{0}^{1}(1-t)^{k} t^{n+1}|d \beta(t)| & =\int_{0}^{1} \sum_{k=i}^{\infty}\left(\begin{array}{c}
k+n \\
n
\end{array}\right)(1-t)^{k} t^{n+1}|d \beta(t)| \\
& \leqq \int_{0}^{1}|d \beta(t)|<\infty
\end{aligned}
$$

hence by (3.1) and integration by parts we obtain

$$
\begin{array}{rl}
\sum_{k=i}^{\infty}\left(\begin{array}{c}
k+n \\
n
\end{array}\right) \int_{0}^{1}(1-t)^{k} t^{n+1} & d \beta(t)=\int_{0}^{1} \sum_{k=i}^{\infty}\left(\begin{array}{c}
k+n \\
n
\end{array}\right)(1-t)^{k} t^{n+1} d \beta(t) \\
= & i \int_{0}^{1} \int_{t}^{1} u^{-1}(1-u)^{-1}\left(\begin{array}{c}
i+n \\
n
\end{array}\right)(1-u)^{i} u^{n+1} d u d \beta(t) \\
= & i \int_{0}^{1} \frac{\beta(u)}{u(1-u)}\left(\begin{array}{c}
i+n \\
n
\end{array}\right)(1-u)^{i} u^{n+1} d u
\end{array}
$$

Denote $\Delta_{n i}=\sum_{k=i}^{\infty}\left(\begin{array}{c}k+n \\ n\end{array}\right) \int_{0}^{1}(1-t)^{k} t^{n+1} d \beta(t)$, then by (3.2)

$$
\sum_{i=0}^{\infty}\left|a_{i}\right|\left|\Delta_{n i}\right| \leqq\left|a_{0}\right|+\sum_{i=1}^{\infty}\left|i a_{i}\right| \int_{0}^{1} \frac{|\beta(u)|}{u(1-u)}\left(\begin{array}{c}
i+n \\
n
\end{array}\right)(1-u)^{i} u^{n+1} d u \text {. }
$$


By (1.3) and Beppo-Levi's theorem

$$
\leqq\left|a_{0}\right|+L \int_{0}^{1} \frac{|\beta(u)|}{u(1-u)} \sum_{i=1}^{\infty}\left(\begin{array}{c}
i+n \\
n
\end{array}\right)(1-u)^{i} u^{n+1} d u
$$

and by (1.6)

$$
=\left|a_{0}\right|+L \int_{0}^{1} \frac{|\beta(u)|}{u(1-u)}\left(1-u^{n+1}\right) d u<\infty
$$

Therefore we have proved the existence of $S_{n}^{*}(\beta)$ for every $n \geqq 0$.

Now

$$
S_{n}^{*}(\beta)-s_{m}=\sum_{i=0}^{\infty} a_{i} \Delta_{n i}-\sum_{i=0}^{m} a_{i}=-\sum_{i=0}^{m} a_{i}\left(1-\Delta_{n i}\right)+\sum_{i=m+1}^{\infty} a_{i} \Delta_{n i} .
$$

By Agnew's theorem [1] we have to show that when $n \equiv n(\lambda)$ and $m \equiv m(\lambda)$

$$
\lim _{\lambda \rightarrow \infty} \frac{1}{i} \sum_{k=0}^{i-1}\left(\begin{array}{c}
k+n \\
n
\end{array}\right) \int_{0}^{1}(1-t)^{k} t^{n+1} d \beta(t)=0 \text { for } i=1,2, \cdots
$$

and

$$
\limsup _{\lambda \rightarrow \infty}\left\{\sum_{i=1}^{m} \frac{1}{i}\left|1-\Delta_{n i}\right|+\sum_{i=m+1}^{\infty} \frac{1}{i}\left|\Delta_{n i}\right|\right\}=A_{q}
$$

Now (3.4) follows by (1.5) in the same manner in which the regularity of $\left(S^{*}, \mu\right)$ is proved (see [7] \$4). In a way similar to the proof of (3.2) we obtain

$$
\frac{1}{i} \sum_{k=0}^{i-1}\left(\begin{array}{c}
k+n \\
n
\end{array}\right) \int_{0}^{1}(1-t)^{k} t^{n+1} d \beta(t)=\int_{0}^{1} \frac{1-\beta(u)}{u(1-u)}\left(\begin{array}{c}
i+n \\
n
\end{array}\right)(1-u)^{i} u^{n+1} d u,
$$

and thus by (3.2) and (3.6) it follows that

$$
\begin{aligned}
\sum_{i=1}^{m} \frac{1}{i}\left|1-\Delta_{n i}\right|+\sum_{i=m+1}^{\infty} \frac{1}{i}\left|\Delta_{n i}\right|= & \sum_{i=1}^{m}\left|\int_{0}^{1} \frac{1-\beta(u)}{u(1-u)}\left(\begin{array}{c}
i+n \\
n
\end{array}\right)(1-u)^{i} u^{n+1} d u\right| \\
& +\sum_{i=m+1}^{\infty}\left|\int_{0}^{1} \frac{\beta(u)}{u(1-u)}\left(\begin{array}{c}
i+n \\
n
\end{array}\right)(1-u)^{i} u^{n+1} d u\right|
\end{aligned}
$$

For $u \leqq \frac{n}{n+m}$ 


$$
\frac{d}{d u} \sum_{i=1}^{m}\left(\begin{array}{c}
i+n \\
n
\end{array}\right)(1-u)^{i} u^{n}=\sum_{i=1}^{m}\left(\begin{array}{c}
i+n \\
n
\end{array}\right)(1-u)^{i-1} u^{n-1}[(n-(n+i) u] \geqq 0,
$$

and for $1>u \geqq \frac{n+1}{n+m+1}$

$$
\frac{d}{d u} \sum_{i=m+1}^{\infty}\left(\begin{array}{c}
i+n \\
n
\end{array}\right)(1-u)^{i-1} u^{n+1}=\sum_{i=m+1}^{\infty}\left(\begin{array}{c}
i+n \\
n
\end{array}\right)(1-u)^{i-2} u^{n}[n+1-(n+i) u] \leqq 0 .
$$

Since $m(\lambda) / n(\lambda) \rightarrow q$ as $\lambda \rightarrow \infty$, for $\lambda \geqq \lambda_{0}$ we have $\frac{n}{n+m} \geqq \frac{1}{2(1+q)}$ and $\frac{n+1}{n+m+1}$ $\leqq \frac{1}{2}\left[\frac{1}{1+q}+1\right]$, consequently for $\lambda \geqq \lambda_{0}$ the functions $u^{-1} \sum_{i=1}^{m}\left(\begin{array}{c}i+n \\ n\end{array}\right)(1-u)^{i} u^{n+1}$ are non-decreasing in $0<u \leqq \frac{1}{2(1+q)}$ and the functions $(1-u)^{-1} \sum_{i=m+1}^{\infty}\left(\begin{array}{c}i+n \\ n\end{array}\right)$ $(1-u)^{i} u^{n+1}$ are non-increasing in $\frac{1}{2}\left[\frac{1}{1+q}+1\right] \leqq u<1$.

Applying the approximation properties of the Bernstein power series of Meyer-König and Zeller [6] we obtain

$$
\lim _{\lambda \rightarrow \infty} \frac{1-\beta(u)}{u(1-u)} \sum_{i=1}^{m}\left(\begin{array}{c}
i+n \\
n
\end{array}\right)(1-u)^{i} u^{n+1}= \begin{cases}\frac{1-\beta(u)}{u(1-u)} & \text { if } \frac{1}{1+q}<u<1 \\
0 \text { if } & 0<u<\frac{1}{1+q}\end{cases}
$$

and the convergence is dominated by the integrable function $K \frac{1-\beta(u)}{1-u}$ for some constant $K$. Similarly

$$
\lim _{\lambda \rightarrow \infty} \frac{\beta(u)}{u(1-u)} \sum_{i=m+1}^{\infty}\left(\begin{array}{c}
i+u \\
n
\end{array}\right)(1-u)^{i} u^{n+1}=\left\{\begin{array}{l}
\frac{\beta(n)}{u(1-u)} \text { if } 0<u<\frac{1}{1+q} \\
0 \text { if } \frac{1}{1+q}<u<1
\end{array}\right.
$$

and the convergence is dominated by the integrable function $H \frac{\beta(u)}{u}$ for some constant $H$.

A proof similar to that of Theorem 2.1 of [3] enables us to conclude that

$$
\lim _{\lambda \rightarrow \infty} \sum_{i=1}^{m}\left|\int_{0}^{1} \frac{1-\beta(u)}{u(1-u)}\left(\begin{array}{c}
i+n \\
n
\end{array}\right)(1-u)^{i} u^{n+1} d u\right|=\int_{1 /(1+q)}^{1} \frac{|1-\beta(u)|}{u(1-u)} d u
$$


and

$$
\lim _{\lambda \rightarrow \infty} \sum_{i=m+1}^{\infty}\left|\int_{0}^{1} \frac{\beta(u)}{u(1-u)}\left(\begin{array}{c}
i+n \\
u
\end{array}\right)(1-n)^{i} u^{n+1} d u\right|=\int_{0}^{1 /(1+q)} \frac{|\beta(u)|}{u(1-u)} d u
$$

This proves (3.5) and completes the proof of our theorem.

REMARK. If $\beta(t)$ is of bounded variation in $[0,1]$ and satisfies

$$
\int_{0}^{1} x^{-1} \int_{0}^{x}|d \beta(u)| d x<\infty
$$

and the series $\sum_{i=0}^{\infty} a_{i}$ satisfies (1.3), then $\left\{S_{n}^{*}(\beta)\right\}$ is the sequence to sequence $\left(S^{*}, \mu\right)$ transform defined by Ramanujan [7] $\$ 4$. This is exactly the case if $\beta(t)$ is non-decreasing in $[0,1]$ and satisfies (1.5) and (1.6).

Proof. By (1.3) and (3.2) for the function $\gamma(u)=\int_{0}^{u}|d \beta(t)|$ we obtain

$$
\begin{aligned}
& \sum_{i=0}^{\infty}\left|a_{i}\right| \sum_{k=i}^{\infty}\left(\begin{array}{c}
k+n \\
n
\end{array}\right) \int_{0}^{1}(1-t)^{k} t^{n+1}|d \beta(t)| \\
& \leqq\left|a_{0}\right|+L \sum_{i=1}^{\infty} \int_{0}^{1} u^{-1}(1-u)^{-1} \int_{0}^{u}|d \beta(t)|\left(\begin{array}{c}
i+n \\
n
\end{array}\right)(1-u)^{i} u^{n+1} d u
\end{aligned}
$$

and applying Beppo-Levi's theorem and (3.7) we get

$$
=\left|a_{0}\right|+L \int_{0}^{1} u^{-1}(1-u)^{-1}\left(1-u^{n+1}\right) \int_{0}^{u}|d \beta(t)| d u<\infty .
$$

Hence

$$
\sum_{i=0}^{\infty} a_{i} \sum_{k=i}^{\infty} \int_{0}^{1}\left(\begin{array}{c}
k+n \\
n
\end{array}\right)(1-t)^{k} t^{n+1} d \beta(t)=\sum_{k=0}^{\infty}\left(\begin{array}{c}
k+n \\
n
\end{array}\right) \int_{0}^{1}(1-t)^{k} t^{n+1} d \beta(t) \sum_{i=0}^{k} a_{i}
$$

where the change of order of summation is justified by (3.8)

$$
=\sum_{k=0}^{\infty}\left(\begin{array}{c}
k+n \\
n
\end{array}\right) \int_{0}^{1}(1-t)^{k} t^{n+1} d \beta(t) s_{k}
$$

This concludes our proof. 
For the proof of Theorem 2 we need the following lemma.

LEMMA. Let $0<\eta<1$ be fixed and suppose that $\beta(t)$ satisfies (1.5) and (1.6). For $0<\delta<\eta$ define

$$
\alpha_{\delta, \eta}^{(\beta)}(t)=\left\{\begin{array}{cl}
\frac{\beta(t)}{t(1-t)} & \delta \leqq t \leqq \eta \\
0 & \text { elsewhere. }
\end{array}\right.
$$

Then for every $\varepsilon>0$ there exists $0<\delta_{0}(\varepsilon)<\eta$ such that for any $\delta, 0<\delta \leqq \delta_{0}$ there exists $n_{0}(\varepsilon, \delta)$ such that

$$
I=\sum_{i=1}^{\infty}\left|\int_{0}^{\eta} \frac{\beta(t)}{t(1-t)}\left(\begin{array}{c}
i+n \\
n
\end{array}\right)(1-t)^{i} t^{n+1} d t-\alpha_{\delta, \eta}^{(\beta)}\left(\frac{n}{i+n}\right) \int_{0}^{1}\left(\begin{array}{c}
i+n \\
n
\end{array}\right)(1-t)^{i} t^{n+1} d t\right|<\varepsilon
$$

provided $n \geqq n_{0}$.

ProOF. Given $\varepsilon>0$ choose $\delta_{0}(\varepsilon)$ such that for any $\delta, 0<\delta \leqq \delta_{0}$ we have

$$
\int_{0}^{\delta} \frac{|\beta(t)|}{t(1-t)} d t<\frac{\varepsilon}{4}
$$

This is possible since $\beta(t)$ satisfies (1. 6 ). Let $\delta, 0<\delta \leqq \delta_{0}$ be fixed, then by (3.10)

$$
\begin{aligned}
0 \leqq I \leqq & \frac{\varepsilon}{4}+\left\{\int_{0}^{\delta}+\int_{\eta}^{1}\right\} \sum_{i=1}^{\infty}\left(\begin{array}{c}
i+n \\
n
\end{array}\right)(1-t)^{i} t^{n+1}\left|\alpha_{\delta, \eta}^{(\beta)}\left(\frac{n}{i+n}\right)\right| d t \\
& +\int_{\delta}^{\eta} \sum_{i=1}^{\infty}\left(\begin{array}{c}
i+n \\
n
\end{array}\right)(1-t) t^{n+1}\left|\frac{\beta(t)}{t(1-t)}-\alpha_{\delta, \eta}^{(\beta)}\left(\frac{n}{i+n}\right)\right| d t \\
& =\frac{\varepsilon}{4}+I_{1}+I_{2} \text {, say. }
\end{aligned}
$$

The Bernstein power series of Meyer-König and Zeller [6] admit the following approximation property. If $f(s)$ is bounded in $[0,1]$, then at each point of continuity, $0<s<1$, of $f(s)$ we have

$$
\lim _{n \rightarrow \infty} \sum_{i=1}^{\infty}\left(\begin{array}{c}
i+n \\
n
\end{array}\right)(1-s)^{i} s^{n+1} f\left(\frac{n}{i+n}\right)=f(s) .
$$

Our $\alpha_{\delta, \eta}^{(\beta)}(t)$ is bounded in $[0,1]$ since $\sup _{0 \leqq t \leqq 1}\left|\alpha_{\delta, \eta}^{(\beta)}(t)\right|=\sup _{\delta \geqq t \leqq \eta}\left|\frac{\beta(t)}{t(1-t)}\right|=M<\infty$. So by 
(3.12) we obtain

(3.13) $\quad \lim \sum_{i=1}^{\infty}\left(\begin{array}{c}i+n \\ n\end{array}\right)(1-t)^{i} t^{n+1} \alpha_{\delta, \eta}^{(\beta)}\left(\frac{n}{i+n}\right)=0$ for $0<t<\delta$ and $\eta<t<1$.

Moreover the convergence in (3.13) is dominated by $M$ whence

$$
\lim _{n \rightarrow \infty} I_{1}=0
$$

For a fixed $t, \delta \leqq t \leqq \eta$ the function $f(s)=\left|\frac{\beta(t)}{t(1-t)}-\alpha_{\delta, \eta}^{(\beta)}(s)\right|$ is bounded for $0 \leqq s$ $\leqq 1$, hence at each point of continuity $\delta \leqq s \leqq \eta$ of $f(s)$ we have by (3.12)

$$
\lim _{n \rightarrow \infty} \sum_{i=1}^{\infty}\left(\begin{array}{c}
i+n \\
n
\end{array}\right)(1-s)^{i} s^{n+1}\left|\frac{\beta(t)}{t(1-t)}-\alpha_{\delta, \eta}^{(\beta)}\left(\frac{n}{i+n}\right)\right|=\left|\frac{\beta(t)}{t(1-t)}-\frac{\beta(s)}{s(1-s)}\right|
$$

For $\delta<s<\eta, f(s)$ is continuous if and only if $\beta(s)$ is continuous, that is almost everywhere in $[\delta, \eta]$ whence by $(3.15)$

$$
\lim _{n \rightarrow \infty} \sum_{i=1}^{\infty}\left(\begin{array}{c}
i+n \\
n
\end{array}\right)(1-t)^{i} t^{n+1}\left|\frac{\beta(t)}{t(1-t)}-\alpha_{\delta, \eta}^{(\beta)}\left(\frac{n}{i+n}\right)\right|=0
$$

almost everywhere in $[\delta, \eta]$. Once again the convergence in (3. 16) is dominated by $2 M$ and so we obtain

$$
\lim _{n \rightarrow \infty} I_{2}=0 .
$$

Combining (3.11), (3.14) and (3.17), our lemma is proved.

ProOF OF THEOREM 2. By (3.2) we obtain

$$
\begin{aligned}
S_{n}^{*}(\beta)-S_{m}^{*}(\gamma)= & \sum_{i=1}^{\infty} i a_{i} \int_{0}^{1}\left[\frac{\beta(u)}{u(1-u)}\left(\begin{array}{c}
i+n \\
n
\end{array}\right)(1-u)^{i} u^{n+1}\right. \\
& \left.-\frac{\gamma(u)}{u(1-u)}\left(\begin{array}{c}
i+m \\
m
\end{array}\right)(1-u)^{i} u^{m+1}\right] d u
\end{aligned}
$$

By Agnew's theorem we have to show that

$$
\begin{aligned}
& \lim _{\lambda \rightarrow \infty} \int_{0}^{1}\left[\frac{\beta(u)}{u(1-u)}\left(\begin{array}{c}
i+n \\
n
\end{array}\right)(1-u)^{i} u^{n+1}-\frac{\gamma(u)}{u(1-u)}\left(\begin{array}{c}
i+m \\
m
\end{array}\right)(1-u)^{i} u^{m+1}\right] d u=0 \\
& \text { for } i=1,2, \cdots,
\end{aligned}
$$


and

$$
\text { (3.19) } \begin{aligned}
\limsup _{\lambda \rightarrow \infty} \sum_{i=1}^{\infty} \mid & \int_{0}^{1}\left[\frac{\beta(u)}{u(1-u)}\left(\begin{array}{c}
i+n \\
n
\end{array}\right)(1-u)^{i} u^{n+1}\right. \\
& \left.-\frac{\gamma(u)}{u(1-u)}\left(\begin{array}{c}
i+m \\
m
\end{array}\right)(1-u)^{i} u^{m+1}\right] d u \mid=B_{q} .
\end{aligned}
$$

Now (3.18) is proved like (3.4) and we have to prove only (3.19). Let $0<\eta<1$ be chosen such that if $\theta=\eta /[q-(q-1) \eta]$, then

$$
\int_{\theta}^{1} \frac{|1-\beta(x)|}{x(1-x)} d x<\varepsilon, \quad \int_{\eta}^{1} \frac{|1-\gamma(x)|}{x(1-x)} d x<\varepsilon
$$

This is possible by (1.6) and the fact that $\theta \rightarrow 1$ as $\eta \rightarrow 1$. By Agnew's theorem

$$
\begin{aligned}
\limsup _{\lambda \rightarrow \infty} \sum_{i=1}^{\infty}\left|\int_{\theta}^{1} \frac{\beta(u)}{u(1-u)}\left(\begin{array}{c}
i+n \\
n
\end{array}\right)(1-u)^{i} u^{n+1} d u-\int_{\eta}^{1} \frac{\gamma(u)}{u(1-u)}\left(\begin{array}{c}
i+m \\
m
\end{array}\right)(1-u)^{i} u^{m+1} d u\right| \\
\quad=\limsup _{\lambda \rightarrow \infty}\left|S_{n(\lambda)}^{*}(\bar{\beta})-S_{m(\lambda)}^{*}(\bar{\gamma})\right|
\end{aligned}
$$

where

$$
\overline{\boldsymbol{\beta}}(t)=\left\{\begin{array}{rll}
\boldsymbol{\beta}(t) & \text { if } & \theta \leqq t \leqq 1 \\
0 & \text { if } & 0 \leqq t<\theta
\end{array}\right.
$$

and

$$
\bar{\gamma}(t)=\left\{\begin{array}{ccc}
\gamma(t) & \text { if } & \eta \leqq t \leqq 1 \\
0 & \text { if } & 0 \leqq t<\eta .
\end{array}\right.
$$

Let $p(\lambda)=\left[\frac{1-\eta}{\eta} m(\lambda)\right]$ (where $[x]$ denotes the largest integer not greater than $x)$, then $p(\lambda) / m(\lambda) \rightarrow \frac{1-\eta}{\eta}$ as $\lambda \rightarrow \infty$ and $p(\lambda) / n(\lambda) \rightarrow \frac{1-\eta}{\eta} q$ as $\lambda \rightarrow \infty$. Now

$$
\left|S_{n}^{*}(\overline{\boldsymbol{\beta}})-S_{m}^{*}(\bar{\gamma}) \leqq\right| S_{n}^{*}(\overline{\boldsymbol{\beta}})-s_{p}|+| s_{p}-S_{m}^{*}(\bar{\gamma}) \mid
$$

and it follows by Theorem 1 and (3.20) that

$$
\begin{aligned}
& \limsup _{\lambda \rightarrow \infty}\left|S_{n}^{*}(\bar{\beta})-S_{m}^{*}(\bar{\gamma})\right| \leqq \limsup _{\lambda \rightarrow \infty}\left|S_{n}^{*}(\bar{\beta})-s_{p}\right|+\limsup _{\lambda \rightarrow \infty}\left|s_{p}-S_{m}^{*}(\bar{\gamma})\right| \\
& =\int_{\theta}^{1} \frac{|1-\beta(x)|}{x(1-x)} d x+\int_{\eta}^{1} \frac{|1-\gamma(x)|}{x(1-x)} d x<2 \varepsilon .
\end{aligned}
$$


Let $\delta>0$ be chosen such that if $\rho=\delta /[q-(q-1) \delta]$, then

$$
\left\{\begin{array}{l}
\int_{0}^{0} \frac{|\beta(t)|}{t(1-t)} d t<\varepsilon, \int_{0}^{\delta} \frac{|\gamma(t)|}{t(1-t)} d t<\varepsilon \\
\limsup _{n \rightarrow \infty} \sum_{i=1}^{\infty}\left|\int_{0}^{\theta}\left(\begin{array}{c}
i+n \\
n
\end{array}\right)(1-t)^{i} t^{n+1} \frac{\beta(t)}{t(1-t)} d t-\alpha_{\rho, \theta}^{(\beta)}\left(\frac{n}{i+n}\right) \int_{0}^{1}\left(\begin{array}{c}
i+n \\
n
\end{array}\right)(1-t)^{i} t^{n+1} d t\right|<\varepsilon \\
\limsup _{m \rightarrow \infty}^{\infty} \sum_{i=1}^{\infty}\left|\int_{0}^{\eta}\left(\begin{array}{c}
i+m \\
m
\end{array}\right)(1-t)^{i} t^{m+1} \frac{\gamma(t)}{t(1-t)} d t-\alpha_{\delta, \eta}^{(\gamma)}\left(\frac{m}{i+m}\right) \int_{0}^{1}\left(\begin{array}{c}
i+m \\
m
\end{array}\right)(1-t)^{i} t^{m+1} d t\right|<\varepsilon
\end{array}\right.
$$

The existence of $\delta$ is guaranteed by our lemma, (1.6) and the fact that $\rho \rightarrow 0$ as $\delta \rightarrow 0$. Denote

$$
\beta_{\rho, \theta}(t)=\left\{\begin{array}{r}
\beta(t) \text { if } \rho \leqq t \leqq \theta \\
0 \text { if elsewhere }
\end{array}\right.
$$

and let $0<t \leqq 1$ be a fixed point of continuity of $\beta_{\rho, \theta}(t)$. For $\zeta>0$ let $\tau(\zeta)>0$ be such that $\left|\beta_{\rho, \theta}(t)-\beta_{\rho, \theta}\left(t_{1}\right)\right|<\zeta$ provided $\left|t-t_{1}\right|<2 \tau$ and $\tau<\frac{\rho}{2}, \tau<\frac{1-\theta}{2}$. For $\lambda \geqq \lambda_{0}$ we have $\left|\frac{m}{n}-q\right|<\frac{2 \tau}{q}$ and $\frac{m}{n}>\frac{q}{2}$ which imply $\mid \frac{n}{i+n}$ $-\frac{m}{q i+m} \mid<\tau$. Now

$$
\begin{aligned}
I & =\sum_{i=1}^{\infty} \frac{\left|\beta_{\rho, \theta}\left(\frac{n}{i+n}\right)-\beta_{\rho, \theta}\left(\frac{m}{q i+m}\right)\right|}{\frac{n}{i+n} \frac{i}{i+n}}\left(\begin{array}{c}
i+n \\
n
\end{array}\right)(1-t)^{i} t^{n+1} \\
& =\left\{\begin{array}{c}
\sum_{i}+\sum_{i}
\end{array}\right\} \frac{\left|\beta_{\rho, \theta}\left(\frac{n}{i+n}\right)-\beta_{\rho, \theta}\left(\frac{m}{q i+m}\right)\right|}{\frac{n}{i+n} \frac{i}{i+n}}\left(\begin{array}{c}
i+n \\
n
\end{array}\right)(1-t)^{i} t^{n+1} \\
& =I_{1}+I_{2}, \text { say. }
\end{aligned}
$$

It follows by (3.12) that 


$$
0 \leqq I_{1} \leqq \frac{8 M}{\rho(1-\theta) \tau^{2}} \sum_{i=0}^{\infty}\left(\frac{n}{i+n}-t\right)^{2}\left(\begin{array}{c}
i+n \\
n
\end{array}\right)(1-t)^{i} t^{n+1} \rightarrow 0 \text { as } n \rightarrow \infty \text {, }
$$

where $M=\sup _{0 \leq t \leq 1}|\beta(t)|$, and

$$
0 \leqq I_{2} \leqq \zeta \frac{4}{\rho(1-\theta)} \sum_{i=0}^{\infty}\left(\begin{array}{c}
i+n \\
n
\end{array}\right)(1-t)^{i} t^{n+1}=\frac{4 \zeta}{\rho(1-\theta)} .
$$

It follows by (3.23) and (3.24) that $0 \leqq \limsup _{\lambda \rightarrow \infty} I \leqq \frac{4 \zeta}{\rho(1-\theta)}$ for every $\zeta>0$, consequently $\lim _{\lambda \rightarrow \infty} I=0$ for every point of continuity $0<t \leqq 1$ of $\beta_{\rho, \theta}(t)$, this is almost everywhere in $[0,1]$, and since the convergence is dominated by $\frac{8 M}{\rho(1-\theta)}$ we obtain

$$
\begin{aligned}
& \lim _{\lambda \rightarrow \infty} \sum_{i=1}^{\infty} \frac{\left|\beta_{\rho, \theta}\left(\frac{n}{i+n}\right)-\beta_{\rho, \theta}\left(\frac{m}{q i+m}\right)\right|}{\frac{n}{i+n} \frac{i}{i+n}} \int^{1}\left(\begin{array}{c}
i+n \\
n
\end{array}\right)(1-t)^{i} t^{n+1} d t \\
& =\lim _{\lambda \rightarrow \infty} \int_{0}^{1}\left[\sum_{i=1}^{\infty} \frac{\left|\beta_{\rho, \theta}\left(\frac{n}{i+n}\right)-\beta_{\rho, \theta}\left(\frac{m}{q i+m}\right)\right|}{\frac{n}{i+n} \frac{i}{i+n}}\left(\begin{array}{c}
i+n \\
n
\end{array}\right)(1-t)^{i} t^{n+1}\right] d t=0 .
\end{aligned}
$$

Since $\int_{0}^{1}\left(\begin{array}{c}i+n \\ n\end{array}\right)(1-t)^{i} t^{n+1} d t=\frac{n+1}{(i+n+1)(i+n+2)}$ we obtain similarly

(3.26) $\lim _{\lambda \rightarrow \infty} \sum_{i=1}^{\infty} \beta_{\rho, \theta}\left(\frac{m}{q i+m}\right)\left|\frac{\int_{0}^{1}\left(\begin{array}{c}i+n \\ n\end{array}\right)(1-t)^{i} t^{n+1} d t}{\frac{n}{i+n} \frac{i}{i+n}}-\frac{\int_{0}^{1}\left(\begin{array}{c}i+m \\ m\end{array}\right)(1-t)^{i} t^{m+1} d t}{\frac{m}{i+m} \frac{i}{i+m}}\right|=0$.

Now $\beta_{\rho, \theta}\left(\frac{m}{q i+m}\right)=\beta_{\rho, \theta}\left(\frac{\frac{m}{i+m}}{q-(q-1) \frac{m}{i+m}}\right)$, so if we denote $\phi(t)=\beta\left(\frac{t}{q-(q-1) t}\right), \quad$ then it follows that $\phi_{\delta, \eta}(t)=\beta_{\rho, \theta}\left(\frac{t}{q-(q-1) t}\right)$ 
and therefore $\alpha_{\delta, \eta}^{(\varphi)}(t)=\frac{\beta_{\rho, \theta}\left(\frac{t}{q-(q-1) t}\right)}{t(1-t)}$. Combining (3.25) and (3.26) we obtain

$$
\begin{gathered}
\lim _{\lambda \rightarrow \infty} \sum_{i=1}^{\infty} \mid \alpha_{\rho, \theta}^{(\beta)}\left(\frac{n}{i+n}\right) \int_{0}^{1}\left(\begin{array}{c}
i+n \\
n
\end{array}\right)(1-t)^{i} t^{n+1} d t \\
-\alpha_{\delta, \eta}^{(\gamma)}\left(\frac{m}{i+m}\right) \int_{0}^{1}\left(\begin{array}{c}
i+m \\
m
\end{array}\right)(1-t)^{i} t^{m+1} d t \mid \\
=\lim _{\lambda \rightarrow \infty} \sum_{i=1}^{\infty}\left|\alpha_{\delta, \eta}^{(\varphi)}\left(\frac{m}{i+m}\right)-\alpha_{\delta, \eta}^{(\gamma)}\left(\frac{m}{i+m}\right)\right| \int_{0}^{1}\left(\begin{array}{c}
i+m \\
m
\end{array}\right)(1-t)^{i} t^{m+1} d t \\
=\lim _{\lambda \rightarrow \infty} \int_{0}^{1} \sum_{i=1}^{\infty} \mid \alpha_{\delta, \eta}^{(\varphi)}\left(\frac{m}{i+m}\right)-\alpha_{\delta, \eta}^{(\gamma)}\left(\frac{m}{i+m}\right)\left(\begin{array}{c}
i+m \\
m
\end{array}\right)(1-t)^{i} t^{m+1} d t \\
=\int_{0}^{\eta}\left|\beta\left(\frac{t}{q-(q-1) t}\right)-\gamma(t)\right| \\
t(1-t)
\end{gathered}
$$

since by (3.12)

$$
\begin{gathered}
\lim _{\lambda \rightarrow \infty} \sum_{i=1}^{\infty}\left(\alpha_{\delta, \eta}^{(q)}\left(\begin{array}{c}
m \\
i+m
\end{array}\right)-\alpha_{\hat{\delta}, \eta}^{(\gamma)}\left(\frac{m}{i+m}\right)\left(\begin{array}{c}
i+m \\
m
\end{array}\right)(1-t)^{i} t^{m+1}\right. \\
=\left\{\begin{array}{cl}
\alpha_{\delta, \eta}^{(\rho)}(t)-\alpha_{\delta, \eta}^{(\gamma)}(t) & \text { if } \delta \leqq t \leqq \eta \\
0 & \text { elsewhere }
\end{array}\right.
\end{gathered}
$$

almost everywhere in $[0,1]$ and the convergence is dominated. By (3.20), (3.21), (3.22) and (3.27) it follows that

(3.28) $\quad \limsup _{\lambda \rightarrow \infty} \sum_{i=1}^{\infty} \mid \int_{0}^{1}\left[\frac{\beta(u)}{u(1-u)}\left(\begin{array}{c}i+n \\ n\end{array}\right)(1-u)^{i} u^{n+1}\right.$

$$
\left.-\frac{\gamma(u)}{u(1-u)}\left(\begin{array}{c}
i+m \\
m
\end{array}\right)(1-u)^{i} u^{m+1}\right] d u\left|-B_{q}\right| \leqq 8 \varepsilon
$$

Since (3.28) holds for every $\varepsilon>0$, our theorem is proved. 


\section{Proofs of Theorems 3 and 4 .}

Proof of TheOrem 3. By (3.3) and the straightforward identities $a_{n}=\left(1+\frac{1}{n}\right) b_{n}-b_{n-1}(n>0),\left(b_{0}=0\right)$ it follows that

(4. 1) $S_{n}^{*}(\beta)-s_{m}=-\sum_{i=1}^{m}\left[\left(1+\frac{1}{i}\right) b_{i}-b_{i-1}\right]\left(1-\Delta_{n i}\right)+\sum_{i=m+1}^{\infty}\left[\left(1+\frac{1}{i}\right) b_{i}-b_{i-1}\right] \Delta_{n i}$.

Now

$\sum_{i=m+1}^{N}\left[\left(1+\frac{1}{i}\right) b_{i}-b_{i-1}\right] \Delta_{n i}=\sum_{i=m+1}^{N} b_{i}\left[\left(1+\frac{1}{i}\right) \Delta_{n i}-\Delta_{n, i+1}\right]-b_{m} \Delta_{n, m+1}+b_{N} \Delta_{n, N+1}$

and by (1.4), $\lim _{N \rightarrow \infty} b_{N} \Delta_{n, N+1}=0$, hence

$$
\sum_{i=m+1}^{\infty}\left[\left(1+\frac{1}{i}\right) b_{i}-b_{i-1}\right] \Delta_{n i}=\sum_{i=m+1}^{\infty} b_{i}\left[\left(1+\frac{1}{i}\right) \Delta_{n i}-\Delta_{n, i+1}\right]-b_{m} \Delta_{n, m+1}
$$

Thus by (4.1)

$$
\begin{aligned}
S_{n}^{*}(\beta)-s_{m}= & -\sum_{i=1}^{m-1} b_{i}\left[\left(1+\frac{1}{i}\right)\left(1-\Delta_{n i}\right)-\left(1-\Delta_{n, i+1}\right)\right] \\
& -b_{m}\left[\left(1+\frac{1}{m}\right)\left(1-\Delta_{n m}\right)+\Delta_{n, m+1}\right]+\sum_{i=m+1}^{\infty} b_{i}\left[\left(1+\frac{1}{i}\right) \Delta_{n i}-\Delta_{n, i+1}\right] \\
= & \sum_{i=1}^{\infty} \gamma_{n m i} b_{i}, \text { say. }
\end{aligned}
$$

By Agnew's theorem [1] we have to show that

$$
\lim _{\lambda \rightarrow \infty} \gamma_{n m i}=0 \text { for } i=1,2, \cdots
$$

and

$$
\lim _{\lambda \rightarrow \infty} \sum_{i=1}^{\infty}\left|\gamma_{n m i}\right|=C_{q}
$$

Now (4.2) follows exactly as (3.4) and we have to prove (4.3). By (3.6) it follows that for $1 \leqq i \leqq m$

$$
\left(1+\frac{1}{i}\right)\left(1-\Delta_{n i}\right)-\left(1-\Delta_{n, i+1}\right)=\frac{1}{i}\left(1-\Delta_{n i}\right)-\left(\begin{array}{c}
i+n \\
n
\end{array}\right) \int_{0}^{1}(1-t)^{i} t^{n+1} d \beta(t)
$$


404

D. LEVIATAN

$$
\begin{aligned}
& =\int_{0}^{1}\left(\begin{array}{c}
i+n \\
n
\end{array}\right)(1-t)^{i} t^{n+1} d\left\{1-\beta(t)-\int_{t}^{1} \frac{1-\beta(u)}{u(1-u)} d u\right\} \\
& =-\int_{0+}^{1-}\left(\begin{array}{c}
i+n \\
n
\end{array}\right)(1-t)^{i} t^{n+1} d\left\{\int_{t}^{1} \frac{1-u}{u} d\left[\frac{u}{1-u}(1-\beta(u))\right]\right\} .
\end{aligned}
$$

Similarly by (3.2) we obtain for $i \geqq m+1$

$$
\begin{aligned}
\left(1+\frac{1}{i}\right) \Delta_{n i}-\Delta_{n, i+1} & =\frac{1}{i} \Delta_{n i}+\left(\begin{array}{c}
i+n \\
n
\end{array}\right) \int_{0}^{1}(1-t)^{i} t^{n+1} d \beta(t) \\
& =\int_{0+}^{1-}\left(\begin{array}{c}
i+n \\
n
\end{array}\right)(1-t)^{i} t^{n+1} d\left\{\int_{0} \frac{1-u}{u} d\left[\frac{u}{1-u} \beta(u)\right]\right\}
\end{aligned}
$$

Applying the technique we have used in the proof of Theorem 1, it follows by (4.4) that for $\lambda \geqq \lambda_{0}$

$$
\begin{aligned}
& \left|\left(1+\frac{1}{m}\right)\left(1-\Delta_{n m}\right)-\left(1-\Delta_{n, m+1}\right)\right| \\
& \leqq \int_{0}^{1}\left(\begin{array}{c}
m+n \\
n
\end{array}\right)(1-t)^{m} t^{n+1}|d \beta(t)|+\int_{0}^{1}\left(\begin{array}{c}
m+n \\
n
\end{array}\right)(1-t)^{m} t^{n+1} \frac{|1-\beta(t)|}{t(1-t)} d t \\
& \leqq \int_{0}^{1} \sum_{k=[n(q-\varepsilon)]}^{[n(q+\varepsilon)]}\left(\begin{array}{c}
k+n \\
n
\end{array}\right)(1-t)^{k} t^{n+1}|d \beta(t)| \\
& \quad+\int_{0}^{1} \sum_{k=[n(q-\varepsilon)]}^{[n(q+\varepsilon)]}\left(\begin{array}{c}
k+n \\
n
\end{array}\right)(1-t)^{k} t^{n+1} \frac{|1-\beta(t)|}{t(1-t)} d t
\end{aligned}
$$

and as $\beta(t)$ is continuous we obtain

$$
\longrightarrow \int_{1 /(1+q+\varepsilon)}^{1 /(1+q-\varepsilon)}|d \beta(t)|+\int_{1 /(1+q+\varepsilon)}^{1 /(1+q-\varepsilon)} \frac{|1-\beta(t)|}{t(1-t)} d t \quad \text { as } \quad \lambda \longrightarrow \infty .
$$

Having this for every $\varepsilon>0$ it follows by the continuity of $\beta(t)$ that

$$
\lim _{\lambda \rightarrow \infty}\left[\left(1+\frac{1}{m}\right)\left(1-\Delta_{n m}\right)-\left(1-\Delta_{n, m+1}\right)\right]=0 .
$$

Again the technique we have used in the proof of Theorem 1 and a proof 
similar to that of Theorem 2.2 of [3] enable us to conclude that

$$
\lim _{\lambda \rightarrow \infty} \sum_{i=1}^{m-1}\left|\gamma_{n m i}\right|=\int_{1 /(1+q)}^{1-} \frac{1-u}{u}\left|d\left[\frac{u}{1-u}(1-\beta(u))\right]\right|
$$

and

$$
\lim _{\lambda \rightarrow \infty} \sum_{i=m+1}^{\infty}\left|\gamma_{n m i}\right|=\int_{0+}^{1 /(1+q)} \frac{1-u}{u}\left|d\left[\frac{u}{1-u} \beta(u)\right]\right|
$$

Our theorem follows now by (4.6), (4.7) and (4.8).

PROOF of THEOREM 4. The proof is similar to that of Theorem 3, applying Remark 2.1 of [3] instead of Theorem 2.2 of [3]. It remains to prove that for each function $f(t)$ bounded in $[0,1]$, we have at each point $t=x$, $0<x<1$ where $f(x \pm)$ exist,

$$
\lim _{n \rightarrow \infty} \sum_{k=0}^{\infty}\left(\begin{array}{c}
k+n \\
n
\end{array}\right)(1-x)^{k} x^{n+1} f\left(\frac{n}{k+n}\right)=\frac{1}{2}[f(x+)+f(x-)] .
$$

The proof is similar to the proof of the same property for the Bernstein polynomials (see [4] Theorem 1.9.1).

\section{REFERENCES}

[1] R.P. AgNEw, Abel transforms and partial sums of Tauberian series, Ann. of Math., 50(1949), 110-117.

[2] W. BIEGERT, Uber konstanten Tauberscher Art bei den Kreisverfahren der Limitierungstheorie, Dissert. Stuttgart, 1965.

[3] A. JAKIMOVSKI AND D. LEVIATAN, A property of approximation operators and application to Tauberian constants, Math. Z., 102(1967), 177-204.

[4] G. G. LoRENTZ, Bernstein polynomials, Univ. of Toronto press, Toronto, 1953.

[5] W. MEYER-KöNIG Untersuchungen über einige verwandte Limitierungsverfahren, Math. Z., 52(1952), 257-304.

[6] W.MeYer-KöNIG AND K.Zeller, Bernsteinsche Potenzreihen, Studia Math., 19(1960), 89-94.

[ 7 ] M.S. Ramanujan, On Hausdorff and quasi-Hausdorff methods of summbility, Quart. J. Math. Oxford second series, 8(1957), 197-213.

[8] S. SHERIF, A Tauberian constant for the $\left(S, \mu_{n+1}\right)$ transformation, Tôhoku Math. J., 19(1967), 110-125.

\section{DEPARTMENT OF MATHEMATICS}

UNIVERSITY OF ILLINOIS

URBANA, ILLINOIS, U.S.A. 